\title{
BMJ Open Postcholecystectomy diarrhoea rate and predictive factors: a systematic review of the literature
}

\author{
Alexia Farrugia (iD , ${ }^{1,2}$ Joseph Anthony Attard, ${ }^{3}$ Saboor Khan, ${ }^{1}$ Nigel Williams, ${ }^{1}$ \\ Ramesh Arasaradnam ${ }^{2,4}$
}

To cite: Farrugia $A$, Attard JA, Khan S, et al. Postcholecystectomy diarrhoea rate and predictive factors: a systematic review of the literature. BMJ Open 2022;12:e046172. doi:10.1136/ bmjopen-2020-046172

- Prepublication history and additional supplemental material for this paper are available online. To view these files, please visit the journal online (http://dx.doi.org/10.1136/ bmjopen-2020-046172).

Received 24 October 2020 Accepted 15 October 202

\section{Check for updates}

(C) Author(s) (or their employer(s)) 2022. Re-use permitted under CC BY-NC. No commercial re-use. See rights and permissions. Published by BMJ.

${ }^{1}$ General Surgery, University Hospitals Coventry and Warwickshire NHS Trust, Coventry, UK

${ }^{2}$ Biomedical sciences, University of Warwick, Warwick Medical School, Coventry, UK ${ }^{3}$ General Surgery, Lincoln County Hospital, Lincoln, UK ${ }^{4}$ Gastroenterology, University Hospitals Coventry and Warwickshire NHS Trust, Coventry, UK

Correspondence to Dr Ramesh Arasaradnam; r.arasaradnam@warwick.ac.uk

\section{ABSTRACT}

Objectives Cholecystectomy is one of the most common surgical procedures performed worldwide to treat gallstone-related disease. Postcholecystectomy diarrhoea (PCD) is a well-reported phenomenon, however, the actual rate, predictive factors and mechanism of action have not been well determined. A systematic review was undertaken to determine the rate and predictive factors associated with diarrhoea in the postcholecystectomy setting.

Methods The review was conducted according to the Preferred Reporting Items for Systematic Review and Meta-Analysis Protocol. Databases searched included Medline, Embase, Pubmed, Cochrane and Google Scholar up to 29 September 2020. The inclusion criteria consisted of cohort studies or randomised trials which investigated the rate of PCD and predictive factors. Case reports, case series, conference abstracts and expert opinion pieces were excluded as were other systematic reviews as all the original articles from those reviews were included in this review. Papers that did not include PCD as a separate entity were excluded. Bias assessment was performed using the Newcastle-Ottawa Scale for cohort studies and the Cochrane risk of bias tool for randomised controlled trials as appropriate. Data were extracted by two authors (AF and JAA) and an overall rate of PCD was calculated. Predictive factors were also extracted and compared between studies.

Results 1204 papers were obtained and 21 were found to contain relevant information about PCD, including the number of patients developing diarrhoea, method of symptom assessment and time of onset postcholecystectomy. A pooled total of 3476 patients were included across the identified studies with 462 (13.3\%) patients developing PCD. Possible predictive factors varied across all studies, with characteristics such as gender, age and weight of patients postulated as being predictive of PCD, with no agreement across studies.

Discussion PCD is therefore relatively common (13.3\%). This has important implications for patient consent. Patients ought to be investigated early for bile acid diarrhoea in suspected PCD. More studies are required to determine the possible predictive factors for PCD. Limitations of the study included that most studies were not powered for calculation of PCD, and assessment methods between studies varied.

PROSPERO registration number CRD42019140444.

\section{Strengths and limitations of this study}

This review focused on postcholecystectomy diarrhoea and all studies relating to postcholecystectomy symptoms were extensively investigated to extract all possible data.

- Possible predictive factors for postcholecystectomy diarrhoea were assessed which has not been extensively investigated.

- A wide variety of questionnaires was used to assess symptoms making it difficult to standardise postoperative symptoms between studies, and this relied heavily on patient recall thus opening up all the studies to recall bias.

- There was a generally low level of evidence as most studies were cohort studies.

- Patients were followed up for a variety of timeframes across the studies and thus it was difficult to standardise.

\section{INTRODUCTION}

Cholecystectomy is the gold standard treatment for symptomatic gallstone disease, which occurs in up to $22 \%$ of adults. ${ }^{1}$ The laparoscopic approach to this surgery is now well documented and accepted as standard practice, due to the significantly lower morbidity and mortality when compared with to open surgery. $^{2}$ As a result, its adaptation into a laparoscopic procedure has increased the frequency with which cholecystectomy is performed. ${ }^{3} 4$ Despite the notable benefits of cholecystectomy in treating gallstone-related disease, the postoperative course for a proportion of patients may be plagued by persistent or even new symptoms, including new-onset diarrhoea. ${ }^{5}$ This may be distressing for patients and have a significant impact on their quality of life. ${ }^{6}$ While it may be just a minor annoyance for some, others may well consider postcholecystectomy diarrhoea (PCD) to be a social disability. ${ }^{78}$

The actual incidence of PCD is unknown, though there is a wide range reported in the literature $(2.1 \%-57.2 \%) .^{9} 10$ Furthermore, 
implicated mechanisms in the onset of this condition remain significantly under-investigated.

At present, there are two main theories regarding the mechanism. The first suggests changes in the orocaecal and colonic transit times secondary to increased enterohepatic circulation brought on by removal of the gallbladder. ${ }^{7}$ The second mechanism is less well defined and involves the potential role of bile acids in causing diarrhoea. ${ }^{11}$ This mechanism has been proposed in idiopathic bile acid diarrhoea, where there is interruption of a negative feedback loop which controls bile acid synthesis. The working theory is that removal of the gallbladder, thus removing a bile storage system, will lead to over synthesis of bile acids by interrupting the same negative feedback loop, thus causing diarrhoea by overloading the uptake mechanisms in the terminal ileum. ${ }^{12}$ $63.5 \%$ of patients who develop diarrhoea after cholecystectomy develop bile acid diarrhoea. ${ }^{13}$

The aim of this systematic review is to analyse published literature in order to assess the incidence of postcholecystectomy. Potential preoperative factors which may help to predict the development of PCD will also be examined. Recommendations for future direction of research shall be made, if appropriate.

\section{METHODS}

The review was registered on PROSPERO. A literature search was performed on Pubmed, Embase and
Medline, Cochrane, Google Scholar using the keywords 'post-cholecystectomy', 'postoperative', 'cholecystectomy', 'diarrhoea' and 'predictive factors'. There were no language limitations. The last search date was 29 September 2020. There were no restrictions to the year of publication. The search strategy is outlined in figure 1 .

The inclusion criteria were cohort studies or randomised controlled trials (RCTs) which investigated the rate of PCD and predictive factors for this condition. Case reports, case series, conference abstracts and expert opinion pieces were also excluded. Systematic reviews were also excluded as all the original articles from those reviews were included in this review. Studies pertaining to persistent symptoms after laparoscopic cholecystectomy, that is symptoms present preoperatively, rather than new symptoms were also excluded.

Data were extracted from the studies independently and entered into an electronic database. The results were subsequently collated. Data extracted included: patient numbers, age, gender, type of study, indication for surgery, preoperative symptoms, postoperative symptoms, predictive factors. The primary endpoint was to identify the rate of PCD and the secondary endpoint was to identify potential predictive factors.

The systematic review was written according to Preferred Reporting Items for Systematic Reviews and Meta-Analyses guidelines. ${ }^{14}$ Risk of bias assessment was
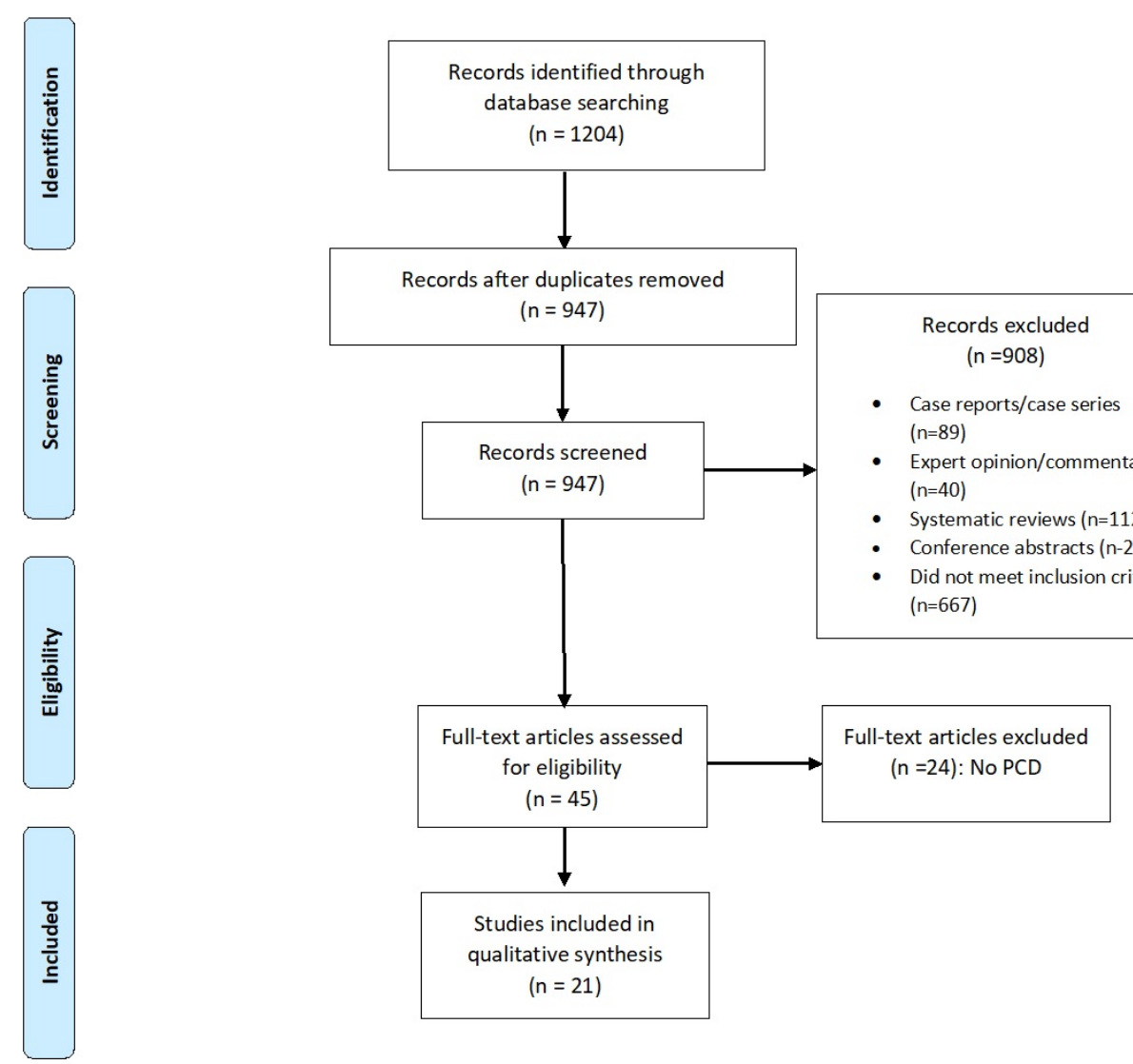

Figure 1 PRISMA flowchart for study selection. PCD, postcholecystectomy diarrhoea; PRISMA, Preferred Reporting Items for Systematic Reviews and Meta-Analyses. 
performed using the Newcastle-Ottawa Scale for cohort studies and the Cochrane risk of bias tool for RCTs as appropriate. ${ }^{1516}$ The papers were classified according to the Oxford OCEBM levels of evidence. ${ }^{17}$

Two independent reviewers (AF and JAA) performed the literature search and reviewed papers for inclusion to ensure the criteria were met. Any differences were resolved by mutual consent. All data extraction was also performed independently by the same two authors.

\section{Patient and public involvement}

There was no patient or public involvement in this study.

\section{RESULTS}

\section{Selected studies}

A total of 1204 papers were identified in the initial search which was reduced to 947 after removal of duplicates. After screening by title and abstract 45 papers were initially considered. Full-text review of these papers revealed that 17 were relevant, that is describing newonset PCD. The reference lists of the chosen articles were also screened, and a further four papers were found to fit the inclusion criteria. This is shown in figure 1. Two articles had to be excluded as full text could not be obtained despite contacting the authors.

\section{Characteristics of included studies}

Most of the studies included were cohort, longitudinal, case-control or cross-sectional studies, of which 11 were prospective and 8 were retrospective. Two studies were RCTs, one of which was an RCT comparing laparoscopic cholecystectomy and cholecystectomy via minilaparotomy, however, one of the reported outcomes was diarrhoea and therefore merited inclusion into this review. ${ }^{18}$ The other RCT was to investigate the effect of Rowachol on post-Laparoscopic cholecystectomy pain, however, the authors also assess symptom clusters including diarrhoea, once again meriting including into the study. ${ }^{10}$ The studies and data obtained are shown in table 1.

\section{Quality assessment and risk of bias}

The Newcastle-Ottawa assessment scale for cohort studies was selected for a risk of bias assessment and adapted to included observational studies. An adaptation of the tool is provided in online supplemental table 1. Patients who underwent laparoscopic cholecystectomy were assessed via a combination of structured interviews and selfreporting. However, as shown in table 1, patient follow-up in a number of studies was not adequate as several patients were not followed up for longer than 3 months. Consequently, this may introduce high levels of bias. Furthermore, lack of a control group in the majority of studies also predisposes to bias in the results. The full risk of bias assessment can be found in online supplemental table 1 . It was not possible to check the heterogeneity of studies due to lack of data, as CIs were not available.

\section{Level of evidence}

The level of evidence was assessed as per the Oxford criteria for Evidence Based medicine. As most of the studies were cohort studies, and a large number of them were retrospective in nature, the general level of evidence was low, classed at 3 or 4 . More detail is shown in online supplemental table 1 and 2.

\section{Demographics}

Demographic data were not routinely available in all studies. However, from those that reported it there were 2250 women and 787 men. Five of the included studies did not provide this information. The age range of patients across the studies was 18-85. One thousand eight hundred and fifty-five cholecystectomies were performed laparoscopically and 378 were open, though once again there were 5 studies where this information was not provided.

\section{Rate of PCD}

A total of 3476 patients were included across all the studies with $462(13.3 \%)$ patients developing PCD, though the rates in the studies vary between $2.1 \%$ and $57.2 \%$. The greater majority of patients were assessed in the first 3-6 months postoperatively, though there is also a large amount of variation in the timing of $\mathrm{PCD}$ as patients were assessed between 6 weeks up to 4 years postoperatively. These are outlined in table 1 . There were not enough data to be able to calculate median time to development of PCD postcholecystectomy.

\section{Predictive factors for PCD}

Several potential risk factors for PCD were identified. Age less than 45 or 50 was mentioned in two studies, as was a high body mass index (BMI). One study suggested that it was commoner in men while two others suggested it was commoner in women. A further two studies associated PCD development with preoperative heartburn or gastritis, while two others still related this to high fat intake. There is lack of consistency in the predictive factors identified in all studies, some studies found no potential predictive factors including sex, age and preoperative symptoms.

\section{DISCUSSION}

Diarrhoea is one of the most reported postoperative symptoms after cholecystectomy, whether this is persistent or new postoperatively, though it varies significantly between studies. ${ }^{1}$ The first mention of this in the literature as a common postoperative sequela is due to Ros and Zambon ${ }^{19}$ who conducted a prospective cohort study to assess postcholecystectomy symptoms. The postoperative assessment took place 2 years after surgery and only 93 of the original 124 patients were available. Eight of these patients reported postoperative loose stools and watery diarrhoea. ${ }^{19}$ In subsequent studies, patients with postcholecystectomy were compared with patients having 


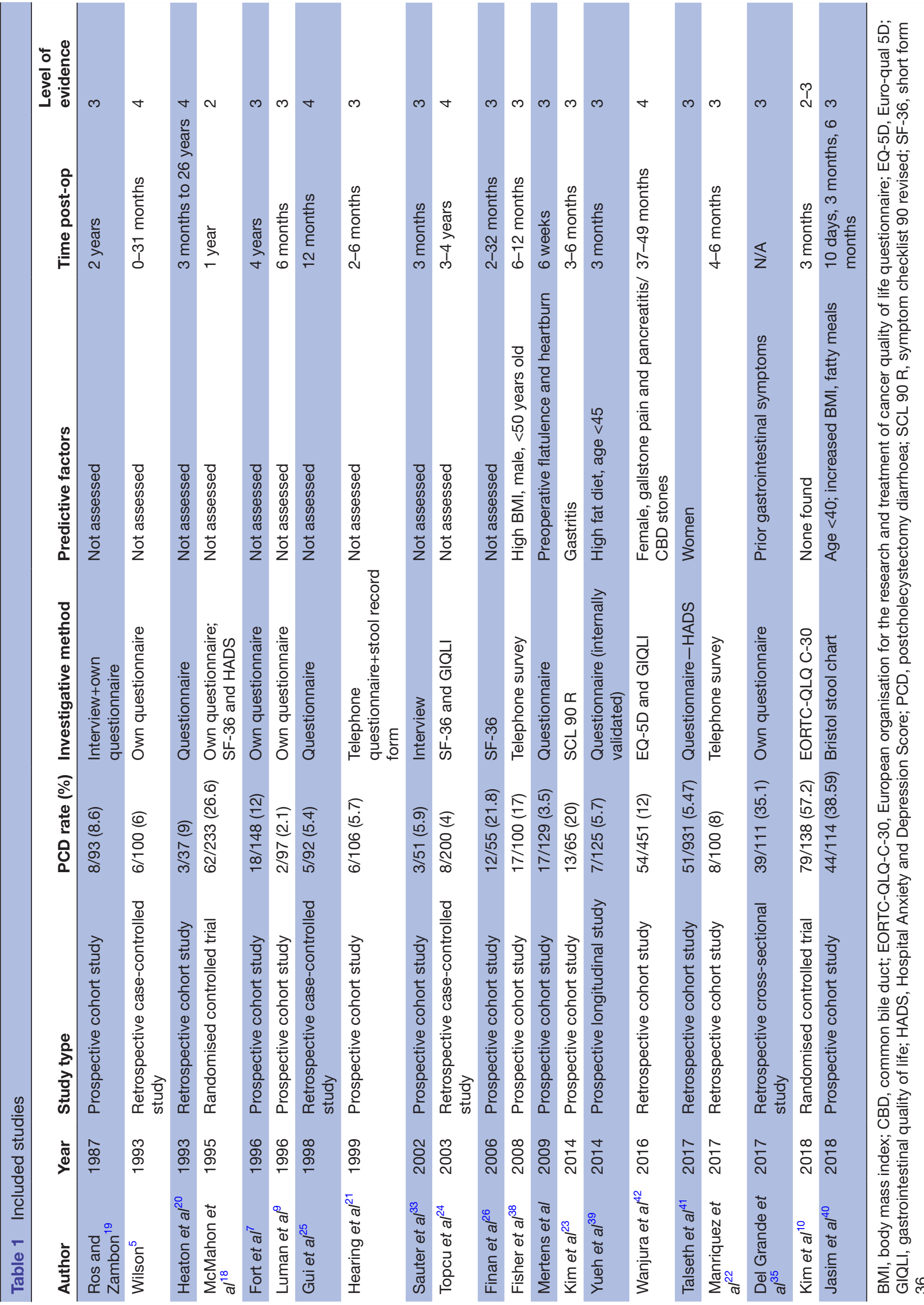

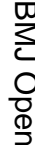

ज

흠

बั

0 
other surgeries such as inguinal hernia, laparoscopic sterilisation and hysterectomy, and bowel habit assessed and compared. ${ }^{5021}$ In some cases, a proportion of patients who developed diarrhoea resolved after a few weeks or months. ${ }^{22} 23$

The question of whether laparoscopic or open cholecystectomy affected the postoperative symptoms was explored. McMahon et al ${ }^{18}$ performed a multicentre RCT to assess the symptomatic outcome between minilaparotomy and laparoscopic cholecystectomy. However, no difference between open or laparoscopic surgery was found. ${ }^{18}$ Topcu et $a l^{24}$ also evaluated gastrointestinal symptoms and quality of life after open and laparoscopic cholecystectomy using the short form 36 (SF-36) and gastrointestinal quality of life (GIQLI) questionnaires, and once again found no difference in the PCD rate. ${ }^{24}$

\section{Investigation of PCD}

A variety of investigative tools including questionnaires (whether previously validated or designed by the researchers), telephone interviews, the Bristol stool chart and stool record forms, from 6 weeks up to 4 years postoperatively ${ }^{912526}$ have been used to assess postcholecystectomy symptoms including diarrhoea. However, this wide range of investigative tools makes study comparison very difficult. In most cases, validated questionnaires were used such as SF36, GIQLI and Gastrointestinal symptom rating scale (GSRS). However, in some studies these were administered retrospectively which limits their objectivity. Some of the questionnaires were also aimed towards general quality of life rather than specific to gastrointestinal symptoms. Other studies used non-validated questionnaires thus limiting their reproducibility. There is also a lot of dependence on patient recall especially in the retrospective studies, as well as differences in describing stool function and what is considered 'diarrhoea' if a standardised tool such as the Bristol stool chart is not used. The main issue with patient recall is the perception of change when change is not always present.

\section{Pathophysiology of PCD and future work for understanding the mechanism}

The concept of PCD and its relationship to bile acids was first mentioned in 1979, where a case series of three patients developing diarrhoea after cholecystectomy showed that two of them had elevated faecal bile acids and in all patients diarrhoea resolved with cholestyramine, thus implying bileacid mediation of such diarrhoea. ${ }^{27}$ Arlow et $a l^{28}$ posited a 'choleric enteropathy' theory when they investigated eight patients with PCD, of whom six had elevated faecal bile acids. They put forward the suggestion that this diarrhoea may be due to the increased production of dihydroxy bile acids and increased daily turnover of primary bile acids due to increase in the enterohepatic cycles as well as continuous bile flux due to a lack of gallbladder. ${ }^{5}$ These patients also responded to cholestyramine therapy. ${ }^{28}$ Fort et al also investigated the prevalence and physiology of PCD. ${ }^{7}$ There is increased bacterial dehydroxylation due to bile acid spending more time in the gut between meals after cholecystectomy ${ }^{29}{ }^{30}$ and the theory that this causes diarrhoea has been put forward, however, it has been shown that the amount of secretion they cause is not enough to cause diarrhoea by Fromm et al. ${ }^{31}$

Intestinal transit after cholecystectomy has been another aspect implicated in PCD. Orocaecal transit has been shown to increase after cholecystectomy, ${ }^{72}$ as is colonic transit though this remains technically within normal limits. ${ }^{7}$ In some cases, though patients did not report diarrhoea after cholecystectomy, they did report an increase in bowel movements and fewer formed stools. ${ }^{334}$ The investigators did not always define what they meant by diarrhoea in a standardised manner (such as number of episodes per day and the use of the Bristol stool chart) and some divided it into 'mild' and 'severe', again without defining what classifies patients into these divisions. ${ }^{23}$ Some papers talk about decrease in stool consistency and increase in bowel motions rather than diarrhoea. ${ }^{35}$ This may tie in with increased Deoxycholic acid (DCA) concentrations after cholecystectomy, however, it was not found to increase basal rectal motility in a study by Edwards $e t a l$, though it was found to increase the sensitivity of the rectum by reducing the volume required to produce a desire to defecate, which may be another way in which DCA can effect postoperative diarrhoea. ${ }^{36}$

Levels of C4, which is a marker of bile acid synthesis, tend to increase after cholecystectomy thus reflecting increased synthesis postoperatively. ${ }^{34}{ }^{37}$ Fibroblast growth factor 19 (FGF19) and C4 levels show significant daily changes and peak at noon, however, after cholecystectomy, this diurnal rhythm changes and FGF19 levels are significantly less at noon, declining at 3 months after surgery. FGF19 levels were shown to correlate to Bile acids (BA) synthesis as measured by C4 levels prior to surgery, but this correlation was lost after cholecystectomy. ${ }^{37}$ Sauter $e t$ $a l$ investigated bile acid malabsorption after cholecystectomy by measuring $\mathrm{C} 4$ levels and investigating changes in bowel habit and found that while most patients describe an increase in bowel motions after cholecystectomy, however, there was no correlation with C4 levels and the described changes in bowel habit, despite an overall increase in C4 levels after cholecystectomy. ${ }^{33}$

Thus, it can be seen that the mechanism behind the development of PCD is still not clearly defined despite several avenues being investigated

\section{Predictive factors for PCD}

Predictive factors identified for PCD varied widely across studies that assessed such factors. Fisher $e t a \hat{l}^{88}$ concluded that it was associated with being men, younger than 50 and having a high BMI, also confirmed by Yueh et $a l^{39}$ and Jasim $e t a t^{40}$ (though in this case the age limit was less than 40 years old) while Del Grande $e t a l^{85}$ associated this with prior gastrointestinal symptoms, though they did not define which ones. ${ }^{358-40}$ Mertens et al clarified this further by stating that it was preoperative flatulence and heartburn which predicted postoperative symptoms including diarrhoea. Yueh $e t a l^{39}$ also found that not following a lowfat diet could be associated with PCD. ${ }^{39}$ Talseth et al study 
found that PCD was more common in patients having cholecystectomy for biliary colic, while Manriquez $e t a l^{22}$ asserted that it was more common in patients having cholecystectomy for asymptomatic cholelithiasis. ${ }^{22}{ }^{41} \mathrm{On}$ the other hand, Kim $e t a l^{10}$ identified no predictive factors including age, BMI, sex, American society of Anaesthetists score, preoperative Endoscopic retrograde cholangiopancreatography (ERCP), comorbidities, difficult laparoscopic cholecystectomy, open conversion or pathology. ${ }^{10}$ Wanjura $e t a t^{42}$ found that several factors were predictive of worse gastrointestinal symptoms after cholecystectomy, including female gender, CBD stones or pancreatitis and gallstone pain as an indication for surgery, however, did not particularly relate this to diarrhoea. ${ }^{42}$ Kim $e t a l^{23}$ also said that gastritis was a preoperative predictive factor for developing post cholecystectomy symptoms, however, once again did not specifically relate this to diarrhoea. ${ }^{23}$

\section{Definition and recommendations for consent and investigation}

The difference in prevalence of diarrhoea across the studies could be attributed to factors such as study design, follow-up length, questionnaire wording (as some studies used non-validated questionnaires), issues with patient recall and definitions of diarrhoea. Unfortunately, most of the studies in this review are not powered specifically to find the rate of PCD, but investigate postcholecystectomy symptoms in general, and in fact most studies focused on dyspeptic symptoms and pain. Some studies were also excluded as they did not specify whether the diarrhoea reported was new onset after cholecystectomy.

There has been no standardised definition of PCD and indeed most of the studies do not specify how they defined 'diarrhoea' in the postoperative period. We feel that a standard definition would be helpful in the investigation of PCD. From the above, we can attempt to define PCD as 'the development of diarrhoea, more than three times a day for more than 4 weeks, post-cholecystectomy'. Investigations for PCD should include basic blood and stool tests, followed by endoscopic examination and ${ }^{75} \mathrm{SeHCAT}$ tests to investigate for inflammatory bowel disease and bile acid diarrhoea respectively. ${ }^{43}$

\section{Possible therapies for PCD}

If it is indeed bile acid diarrhoea, a bile acid sequestrant such as colestyramine could help symptoms. ${ }^{13}$ However, in other patients once other causes have been excluded including inflammatory bowel disease, other symptomatic treamtents are required such as loperamide or dietary modifications. ${ }^{44}$

\section{Strengths and limitations}

The major strength of this review is that we considered the possible predictive factors for the development of PCD. We also looked at all studies involving postcholecystectomy symptoms and if data could be extracted regarding PCD this was also done, thus adding more numbers to the study and providing a more accurate picture of the actual PCD rate. It should also inform the consent process prior to surgery, as currently patients are not always informed that this is a possibility, and may significantly affect their quality of life. ${ }^{45}$

However, there were some methodological limitations. There was no standardisation between studies in terms of follow-up times, as well as questionnaire use. Some authors also used non-validated questionnaires thus making reproducibility difficult. Questionnaires are heavily based on patient recall and there is therefore an element of recall bias in all these studies. Almost all the studies were cohort studies, thus the lack of control group contributed to a low general level of evidence. These limitations could be the reason behind the wide variation of PCD rates across the studies.

\section{Implications for future research}

Larger prospective studies are required to determine the exact rate of PCD and possible predictive factors. It would also be interesting to see how many patients are investigated for PCD using real time clinical data, to investigate how this issue is being handled outside of study protocols. A useful method of keeping better track of such patients is setting up a national registry which could be run by trainees. Another potential method of investigating this would be to set up a large, prospective, national study of patients having cholecystectomy for various reasons, including cholelithiasis, polyps and cancer, and investigate possible predictive factors such as BMI, smoking, sex, age and comorbidities. The quality of life (QOL) preoperatively and postoperatively could also be assessed, especially the difference in QOL between those who develop PCD and those who do not. Further work is also required to determine the exact mechanism behind its development, potentially looking further into the role of FGF19 and C4 levels, and their relationship to bile acid synthesis after cholecystectomy.

\section{CONCLUSION}

PCD is becoming an increasingly recognised issue with an overall incidence of around $13.1 \%$. However, no welldefined predictive factors can be elucidated. It is often not recognised as a problem as patients are not routinely followed up. It is also a significant burden on patients. The mechanism behind its development also needs to be investigated further, though the role of bile acids in this is also becoming more defined. Patients need to be more informed about the possibility of this occurring as part of the consent process preoperatively and in the postoperative period more support needs to be offered to patients in the investigation and diagnosis process.

Contributors AF and JAA wrote the manuscript. SK, NW and RA supervised the writing, helped with analysis and led the research project. RA acting as Guarantor.

Funding This research was supported by Bowel Research UK.

Competing interests None declared.

Patient consent for publication Not applicable.

Provenance and peer review Not commissioned; externally peer reviewed.

Data availability statement All data relevant to the study are included in the article or uploaded as supplementary information. 
Supplemental material This content has been supplied by the author(s). It has not been vetted by BMJ Publishing Group Limited (BMJ) and may not have been peer-reviewed. Any opinions or recommendations discussed are solely those of the author(s) and are not endorsed by BMJ. BMJ disclaims all liability and responsibility arising from any reliance placed on the content. Where the content includes any translated material, BMJ does not warrant the accuracy and reliability of the translations (including but not limited to local regulations, clinical guidelines, terminology, drug names and drug dosages), and is not responsible for any error and/or omissions arising from translation and adaptation or otherwise.

Open access This is an open access article distributed in accordance with the Creative Commons Attribution Non Commercial (CC BY-NC 4.0) license, which permits others to distribute, remix, adapt, build upon this work non-commercially, and license their derivative works on different terms, provided the original work is properly cited, appropriate credit is given, any changes made indicated, and the use is non-commercial. See: http://creativecommons.org/licenses/by-nc/4.0/.

\section{ORCID iD}

Alexia Farrugia http://orcid.org/0000-0002-4366-9068

\section{REFERENCES}

1 Lamberts MP, Lugtenberg M, Rovers MM, et al. Persistent and de novo symptoms after cholecystectomy: a systematic review of cholecystectomy effectiveness. Surg Endosc 2013;27:709-18.

2 Mclntyre RC, Zoeter MA, Weil KC, et al. A comparison of outcome and cost of open vs. laparoscopic cholecystectomy. J Laparoendosc Surg 1992;2:143-8.

3 van Dijk AH, van Roessel S, de Reuver PR, et al. Systematic review of cystic duct closure techniques in relation to prevention of bile duct leakage after laparoscopic cholecystectomy. World J Gastrointest Surg 2018;10:57-69.

4 O'DONNELL LJD. Post-Cholecystectomy diarrhoea: a running commentary. Gut 1999;45:796-7.

5 Wilson RG, Macintyre IMC. Symptomatic outcome after laparoscopic cholecystectomy. Br J Surg 2005;80:439-41.

6 Farahmandfar MR, Chabok M, Alade M, et al. Post cholecystectomy Diarrhoea-A systematic review. Surg Sci 2012;03:332-8.

7 Fort JM, Azpiroz F, Casellas F, et al. Bowel habit after cholecystectomy: physiological changes and clinical implications. Gastroenterology 1996;111:617-22.

8 GBDDD C. Estimates of global, regional, and national morbidity, mortality, and aetiologies of diarrhoeal diseases: a systematic analysis for the global burden of disease study 2015. The Lancet Infectious diseases 2017;17:909-48.

9 Luman W, Adams WH, Nixon SN, et al. Incidence of persistent symptoms after laparoscopic cholecystectomy: a prospective study. Gut 1996;39:863-6.

$10 \mathrm{Kim} \mathrm{H}$, Han IW, Heo JS, et al. Postcholecystectomy syndrome: symptom clusters after laparoscopic cholecystectomy. Annals of Surgical Treatment and Research 2018;95:135-40.

11 Appleby RN, Nolan JD, Johnston IM, et al. Novel associations of bile acid diarrhoea with fatty liver disease and gallstones: a cohort retrospective analysis. BMJ Open Gastroenterology 2017;4:e000178.

12 Walters JRF, Tasleem AM, Omer OS, et al. A new mechanism for bile acid diarrhea: defective feedback inhibition of bile acid biosynthesis. Clinical Gastroenterology and Hepatology 2009;7:1189-94.

13 Ruiz-Campos L, Gisbert JP, Ysamat M, et al. Systematic review with meta-analysis: the prevalence of bile acid malabsorption and response to colestyramine in patients with chronic watery diarrhoea and previous cholecystectomy. Aliment Pharmacol Ther 2019;49:242-50.

14 Moher D, Liberati A, Tetzlaff J, et al. Preferred reporting items for systematic reviews and meta-analyses: the PRISMA statement. PLoS Med 2009;6:e1000097.

15 GSe HJPT. Cochrane Handbook for Systematic Reviews of Interventions Version 5.1.0 [updated March 2011]. : The Cochrane Collaboration, 20112011.

16 Wells GA SB, O'Connell D, Peterson J. The Newcastle Ottawa scale (NOS) for assessing the quality of nonrandomised studies in metaanalyses. Ottawa: Ottawa Hospital Research Institute, 2000. http:// www.ohri.ca/programs/clinical_epidemiology/oxford.asp

17 Working Group Oxford Centre for Evidence-based Medicine. OCEBM levels of evidence, 2009. Available: https://www.cebm.ox.ac.uk/ resources/levels-of-evidence/ocebm-levels-of-evidence
18 McMahon AJ, Ross S, Baxter JN, et al. Symptomatic outcome 1 year after laparoscopic and minilaparotomy cholecystectomy: a randomized trial. Br J Surg 2005;82:1378-82.

19 Ros E, Zambon D. Postcholecystectomy symptoms. A prospective study of gall stone patients before and two years after surgery. Gut 1987;28:1500-4.

20 Heaton KW, Parker D, Cripps $\mathrm{H}$. Bowel function and irritable bowe symptoms after hysterectomy and cholecystectomy--a population based study. Gut 1993;34:1108-11.

21 Hearing SD, Thomas LA, Heaton KW, et al. Effect of cholecystectomy on bowel function: a prospective, controlled study. Gut 1999;45:889-94.

22 Manríquez E, Tejos R, Rojas A. Postcholecystectomy diarrhea is a frequent problem? Revista Chilena de Cirugia 2017;69:376-81.

$23 \mathrm{Kim} \mathrm{GH}$, Lee HD, Kim M, et al. Fate of dyspeptic or colonic symptoms after laparoscopic cholecystectomy. J Neurogastroenterol Motil 2014;20:253-60.

24 Topçu O, Karakayali F, Kuzu MA, et al. Comparison of long-term quality of life after laparoscopic and open cholecystectomy. Surg Endosc 2003;17:291-5.

25 Gui GP, Cheruvu CV, West N, et al. Is cholecystectomy effective treatment for symptomatic gallstones? clinical outcome after longterm follow-up. Ann R Coll Surg Engl 1998;80:25-32.

26 Finan KR, Leeth RR, Whitley BM, et al. Improvement in gastrointestinal symptoms and quality of life after cholecystectomy. The American Journal of Surgery 2006;192:196-202.

27 Hutcheon DF, Bayless TM, Gadacz TR. Postcholecystectomy diarrhea. JAMA 1979;241:823-4.

28 Arlow FL, Dekovich AA, Priest RJ. Bile Acid-Mediated Postcholecystectomy diarrhea. Arch Intern Med 1987;147:1327-9.

29 Turumin JL, Shanturov VA, Turumina HE. The role of the gallbladder in humans. Revista de Gastroenterología de México 2013;78:177-87.

30 Phillips SF. Diarrhea after cholecystectomy: if so, why? Gastroenterology 1996;111:816-8.

31 Fromm H, Tunuguntla AK, Malavolti M, et al. Absence of significant role of bile acids in diarrhea of a heterogeneous group of postcholecystectomy patients. Dig Dis Sci 1987;32:33-44.

32 Penagini R, Spiller RC, Misiewicz JJ, et al. Effect of cholecystectomy on mouth-to-cecum transit of a liquid meal. Dig Dis Sci 1988;33:19-22.

33 Sauter GH, Moussavian AC, Meyer G, et al. Bowel habits and bile acid malabsorption in the months after cholecystectomy. Am J Gastroenterol 2002;97:1732-5.

34 Moussavian CA, Parhofer K, Juengst D. Effect of cholecystectomy on bile acid metabolism and serum lipoprotein profiles. Gastroenterology 2000;118:A884.

35 Del Grande LdeM, Leme LFP, Marques FP, et al. Prevalence and predictors of changes in bowel habits after laparoscopic cholecystectomy. ABCD. Arquivos Brasileiros de Cirurgia Digestiva 2017;30:3-6.

36 Edwards CA, Brown S, Baxter AJ, et al. Effect of bile acid on anorectal function in man. Gut 1989;30:383-6.

37 Barrera F, Azócar L, Molina H, et al. Effect of cholecystectomy on bile acid synthesis and circulating levels of fibroblast growth factor 19 . Ann Hepatol 2015;14:710-21.

38 Fisher M, Spilias DC, Tong LK. Diarrhoea after laparoscopic cholecystectomy: incidence and main determinants. ANZ J Surg 2008;78:482-6.

39 Yueh T-P, Chen F-Y, Lin T-E, et al. Diarrhea after laparoscopic cholecystectomy: associated factors and predictors. Asian Journal of Surgery 2014;37:171-7.

40 Jasim H. Postcholecystectomy bowel motion changes and diarrhoea: a prospective study. Iraqi Medical Journal 2018;64:9-15 https://www. researchgate.net/publication/325396721_Postcholecystectomy_ bowel_motion_changes_and_diarrhea_a_prospective_study

41 Talseth A, Edna T-H, Hveem K, et al. Quality of life and psychological and gastrointestinal symptoms after cholecystectomy: a populationbased cohort study. BMJ Open Gastroenterology 2017;4:e000128.

42 Wanjura V, Sandblom G. How do quality-of-life and gastrointestinal symptoms differ between post-cholecystectomy patients and the background population? World J Surg 2016;40:81-8.

43 Arasaradnam RP, Brown S, Forbes A. Guidelines for the investigation of chronic diarrhoea in adults: British Society of gastroenterology. 3 edn. Gut, 2018.

$44 \mathrm{Li}$ Z, Vaziri H. Treatment of chronic diarrhoea. Best Pract Res Clin Gastroenterol 2012;26:677-87.

45 Hussain A, Verzune M, Azhar MS, Saad Azhar M. Diarrhoea after laparoscopic cholecystectomy: current consenting practice inappropriate. Int J Surg 2016;36:S120. 